\title{
BMJ Open Lifetime risk of prostate cancer overdiagnosis in Australia: quantifying the risk of overdiagnosis associated with prostate cancer screening in Australia using a novel lifetime risk approach
}

Thanya Pathirana, ${ }^{\odot, 2}$ Andrew Hayen, ${ }^{3}$ Jenny Doust, ${ }^{1}$ Paul Glasziou, ${ }^{1}$ Katy Bell ${ }^{\odot, 4}$

To cite: Pathirana T, Hayen A, Doust J, et al. Lifetime risk of prostate cancer overdiagnosis in Australia: quantifying the risk of overdiagnosis associated with prostate cancer screening in Australia using a novel lifetime risk approach. BMJ Open 2019;9:e022457. doi:10.1136/ bmjopen-2018-022457

- Prepublication history and additional material for this paper are available online. To view these files, please visit the journal online (http://dx.doi. org/10.1136/bmjopen-2018022457).

Received 3 March 2018 Revised 21 October 2018 Accepted 17 January 2019

Check for updates

(C) Author(s) (or their employer(s)) 2019. Re-use permitted under CC BY-NC. No commercial re-use. See rights and permissions. Published by BMJ.

For numbered affiliations see end of article.

Correspondence to Dr Thanya Pathirana; thanindu@gmail.com

\section{ABSTRACT}

Objectives To quantify the risk of overdiagnosis associated with prostate cancer screening in Australia using a novel lifetime risk approach.

Design Modelling and validation of the lifetime risk method using publicly available population data.

Setting Opportunistic screening for prostate cancer in the Australian population.

Participants Australian male population (1982-2012). Interventions Prostate-specific antigen testing for prostate cancer screening.

Primary and secondary outcome measures Primary: lifetime risk of overdiagnosis in 2012 (excess lifetime cancer risk adjusted for changing competing mortality); Secondary: lifetime risk of prostate cancer diagnosis (unadjusted and adjusted for competing mortality); Excess lifetime risk of prostate cancer diagnosis (for all years subsequent to 1982).

Results The lifetime risk of being diagnosed with prostate cancer increased from $6.1 \%$ in 1982 ( 1 in 17) to $19.6 \%$ in 2012 (1 in 5). Using 2012 competing mortality rates, the lifetime risk in 1982 was $11.5 \%$ (95\% Cl 11.0\% to $12.0 \%)$. The excess lifetime risk of prostate cancer in 2012 (adjusted for changing competing mortality) was $8.2 \%$ (95\% Cl $7.6 \%$ to $8.7 \%$ ) (1 in 13). This corresponds to $41 \%$ of prostate cancers being overdiagnosed.

Conclusions Our estimated rate of overdiagnosis is in agreement with estimates using other methods. This method may be used without the need to adjust for lead times. If annual (cross-sectional) data are used, then it may give valid estimates of overdiagnosis once screening has been established long enough for the benefits from the early detection of non-overdiagnosed cancer at a younger age to be realised in older age groups.

\section{INTRODUCTION}

After the commencement of population cancer screening, a spike in the apparent incidence of the cancer is expected. These excess cancers may reflect both (1) detection of cancers that otherwise would have progressed
Strengths and limitations of this study

- We describe a new method which may be easily applied using freely available statistical software and publicly available administrative datasets.

- The method may be used in combination with other methods to triangulate the likely extent of cancer overdiagnosis in populations where data from randomised controlled trials are not available.

- Unlike other methods that use observational data to estimate overdiagnosis, there is no need to adjust for the lead time advancement in the time of diagnosis for non-overdiagnosed cancers.

- However, when using cross-sectional data for this method, screening needs to be established for a period at least as long as the average lead time of non-overdiagnosed cancers.

- The method also assumes that there are no underlying changes in risk factors for the cancer over time.

to symptomatic disease (non-overdiagnosed cancers; potential beneficial effect of screening) and (2) detection of cancers that otherwise would not have become symptomatic (overdiagnosed cancers; potential harmful effect of screening). Histopathology assessment of screen-detected abnormalities may result in overdiagnosis due to imperfect discrimination between biologically benign lesions and lesions that would progress towards clinical malignancy if left untreated. ${ }^{1}$ As there is no direct way to predict with certainty which individual cancers will progress without treatment and which will not, we must use indirect population-based methods to estimate the proportion of cancers that are likely to have been overdiagnosed.

Estimations of the extent of overdiagnosis need to account for lead time, the time 
between when a non-overdiagnosed cancer is diagnosed due to early detection through screening, and when it would have been detected clinically. Compared with the unscreened group, lead time causes an increase in the annual cancer incidence during screening and a compensatory drop in the annual incidence after screening ends. This occurs until the end of the longest lead time of a non-overdiagnosed cancer. ${ }^{2}$ Current methods to quantify cancer overdiagnosis from screening require estimates of lead time (s).$^{2-4}$ However, lead time estimation is statistically problematic, ${ }^{5}$ as assumptions may result in either overestimation of the maximum lead time and underestimation of overdiagnosis ${ }^{6-8}$ or underestimation of the maximum lead time and overestimation of overdiagnosis. ${ }^{910}$ Hence, methods that sidestep explicit assumptions about the lead time would be useful.

\section{LIFETIME RISK OF CANCER IN TRIALS}

One alternative method that would not require assumptions about the lead time distribution for progressive cancers is a post-trial study after a screening randomised controlled trial (RCT), with follow-up continuing until all trial participants have died. We could then calculate the lifetime incidence of a cancer diagnosis for the screened and unscreened trial arms: any excess incidence in the screened arm would be attributable to overdiagnosis. This is essentially the excess cumulative incidence method after a stop-screen trial ${ }^{41-13}$ using an extreme follow-up time to ensure that the longest possible lead time for any screen-detected cancers is captured. Challenges to this method include the long time frame, the need for collaboration with trialists to access individual patient data for post-trial linkage to cancer registries and mortality data, and losses to follow-up due to emigration or other causes. There may also be issues of reduced applicability of overdiagnosis estimates from older trials to the present situation, for example, due to changes in screening and/or diagnostic test thresholds and technologies, as well as changes in the effectiveness of treatments that may change the effectiveness of screening and changes in competing mortality risk. However, the most important challenge may be post-trial screening in both the intervention and control arms-if this is measured then it may be possible to adjust for this statistically, but all post-trial studies to date have only measured screening during the within trial period.

\section{USING POPULATION DATA TO ESTIMATE LIFETIME RISK OF CANCER}

An observational alternative to estimating lifetime risk of a cancer in trials is to apply life table and related methods to annual population data. These methods are currently used by cancer agencies worldwide to calculate lifetime risks of being diagnosed with cancer and of dying from cancer, which are commonly framed in terms of a 'one in x' chance to communicate the risk in a more readily understood way (eg, women have a one in eight chances of being diagnosed with breast cancer in their lifetime). ${ }^{14}$ Life table methods commonly use cross-sectional age-specific cancer incidence and mortality rates, together with all-cause mortality rates, to estimate lifetime and age-specific conditional probabilities of developing a particular cancer in a hypothetical cohort who experience each age-specific rate over their lifetimes.

Statistical methods have been developed to estimate these probabilities where rates are reported per personyears alive (including people who are living with a cancer diagnosis; this is how these data are commonly reported in cancer registries) and to comprehensively account for competing risks. ${ }^{15}$ This method is used in the DevCan software provided free of charge on the Surveillance, Epidemiology, and End Results program website, ${ }^{16}$ which we used in the example of prostate cancer in Australian men below. As well as illustrating how the life table method may be used to estimate lifetime risk of overdiagnosis, to our knowledge these results are the first estimates of the extent of prostate cancer overdiagnosis among Australian men.

\section{USING POPULATION DATA TO ESTIMATE LIFETIME RISK OF OVERDIAGNOSIS}

If screening activities have been in place for a sufficiently long time, then a comparison of lifetime risks of cancer diagnosis, using annual population data before and after the introduction of screening, may be used to estimate cancer overdiagnosis. However, as reductions in competing mortality over time can increase lifetime cancer risks, lifetime risks that are calculated using contemporaneous competing mortality rates cannot be simply compared. For example, decreasing competing mortality from cardiovascular disease may lead to increasing cancer risk because more people live long enough for cancers to present clinically. To ensure that a difference in lifetime risk is due to overdiagnosis rather than changes in competing mortality, we aimed to calculate the lifetime risk of overdiagnosis using the same competing mortality risk for both years before and after the introduction of screening.

\section{LIFETIME RISK OF PROSTATE CANCER OVERDIAGNOSIS IN AUSTRALIAN MEN}

In our example on prostate cancer overdiagnosis, we set competing mortality to the rates reported for the most recent calendar year (2012, 'adjusted lifetime risk') by using the age-specific prostate cancer mortality rates and age-specific all-cause mortality rates for 2012, while using the age-specific incidence rates for the index year. We then estimated the excess lifetime risk as the difference between the adjusted lifetime risk of being diagnosed with cancer in an index year (after screening was introduced) and the adjusted lifetime risk of cancer diagnosis in the reference year (before screening was introduced). 


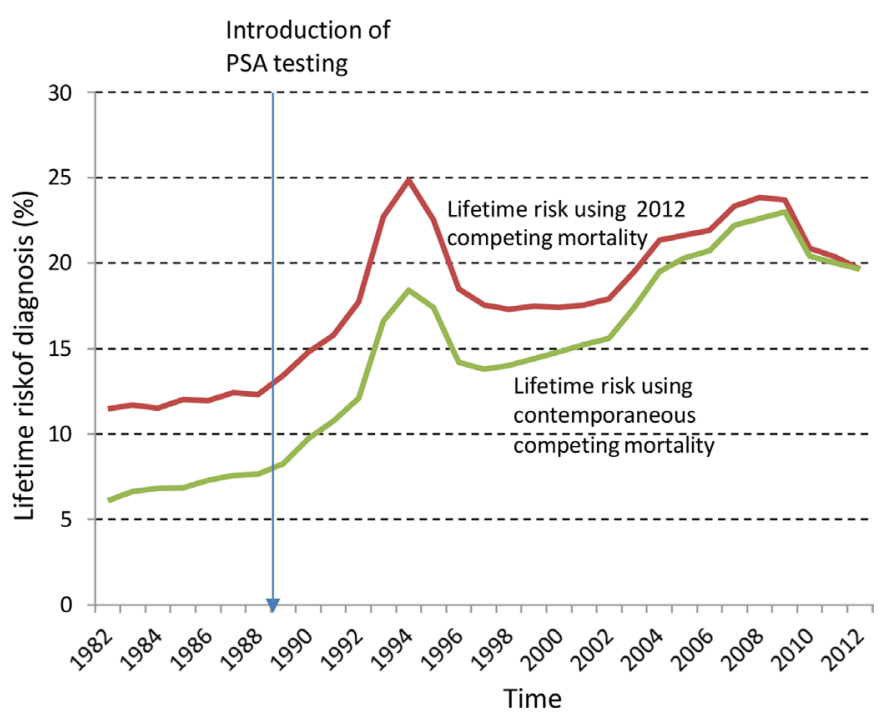

Figure 1 Lifetime risk of prostate cancer diagnosis in Australia from 1982 to 2012. PSA, prostate-specific antigen.

In DevCan, the probability of getting a first cancer diagnosis in a specific age interval is estimated based on a conditional cumulative incidence function using: first incidence of cancer per person-years alive, rate of cancer deaths per person-years alive and the rate of other (non-cancer) deaths per person-years alive. ${ }^{15} 17$ Lifetime risk of overdiagnosis was calculated as a proportion of the (adjusted) lifetime risk in an unscreened population or in a screened population. In the previous literature, the results for the screened population have been suggested as the most helpful presentation. ${ }^{318} 19$

\section{Prostate-specific antigen test and incidence of prostate cancer}

The prostate-specific antigen (PSA) test, which first received government subsidy in Australia in 1989, is used to screen for prostate cancer or for post-treatment surveillance. The increasing incidence of prostate cancer and lifetime risk of being diagnosed is thought by many to be due to PSA testing. ${ }^{2021}$ Despite some decline in the rate of PSA screening in recent years, approximately 15\%-18\% of men in the 55-65 age group had a PSA test in $2015 .^{22}$

\section{Data}

Data for age-specific prostate cancer incidence and mortality, and all-cause mortality were retrieved for the years 1982-2012 (most recent available) from Australian Cancer Database (ACD) compiled by the Australian Institute of Health and Welfare (AIHW) based on data provided by the state and territory cancer registries. By legislation, cancer is a notifiable disease in all Australian states and territories. Various institutions, such as hospitals, pathology laboratories and registries of births, deaths and marriages, are required to report cancer cases and deaths to their jurisdictional cancer registry. Each registry will then supply incidence data annually to the AIHW. These data are checked, standardised and compiled into the ACD, the only repository of national cancer incidence data. The data from each of Australia's cancer registries are classified by International Agency for Research on Cancer as 'A', which is the highest data quality grade in their scale. ${ }^{23}$ Mortality data were derived from the National Mortality Database, AIHW. Cause of Death Unit Record File data are provided to the AIHW by the Registries of Births, Deaths and Marriages and the National Coronial Information System and include cause of death coded by the Australian Bureau of Statistics. Thus, these are routinely collected, annual, administrative population data. ${ }^{21} 24$ Therefore, our calculations included populations where screening is available or offered and not limited to just the men who actually underwent screening but those who were diagnosed through all means.

We used DevCan V.6.7.5 statistical software provided by US National Cancer Institute ${ }^{16}$ to generate life tables and calculate lifetime risks of developing prostate cancer for each calendar year. We first calculated the rates adjusting for contemporaneous competing mortality, and then calculated adjusted rates using 2012 competing mortality for all years. To evaluate whether screening was established for a sufficiently long time to make the assumption of the method tenable, we examined temporal trends in PSA use for screening based on claims made to the Medicare Benefits Schedule (MBS). Finally, as a sensitivity analysis, we calculated excess lifetime risk using birth cohort (longitudinal) data rather than annual (cross-sectional) data.

\section{Patient and public involvement}

As this was a secondary analysis of publicly available population data, no informed consent of patients was required for this study.

\section{RESULTS}

In the late 1980 s, there was a rapid increase in lifetime risk of being diagnosed with prostate cancer (figure 1). Although there was some increase in lifetime risk before the introduction of PSA testing to Australia in 1989, the steepest rise appears after 1989. The lifetime risk of being diagnosed with prostate cancer increased from $6.1 \%$ in 1982 ( 1 in 17 ) to $18.4 \%$ in 1994 (1 in 6) (online supplementary table 1). Using 2012 competing mortality rates for all years, the lifetime risks for all years were higher, but mirrored the changes described above. Adjusted lifetime risk increased from $11.5 \%(11.0 \%-12.0 \%)$ in 1982 (1 in 9) to reach a peak of $24.8 \%(24.4 \%-25.4 \%)$ in 1994 (1 in 4 ). The lifetime risk of prostate cancer has shown some fluctuations after this, but has never dipped below $15 \%$, and in the most recent year (2012) was $19.7 \%$ (95\% CI $19.4 \%$ to $20.0 \%$ ) ( 1 in 5 ).

The excess in adjusted lifetime risk of each index year compared with 1982 also parallels the temporal trends described above (figure 2). Once screening is established in the population, the excess adjusted lifetime cancer risk represents potential overdiagnosis; in our example, we assumed establishment of screening in the 


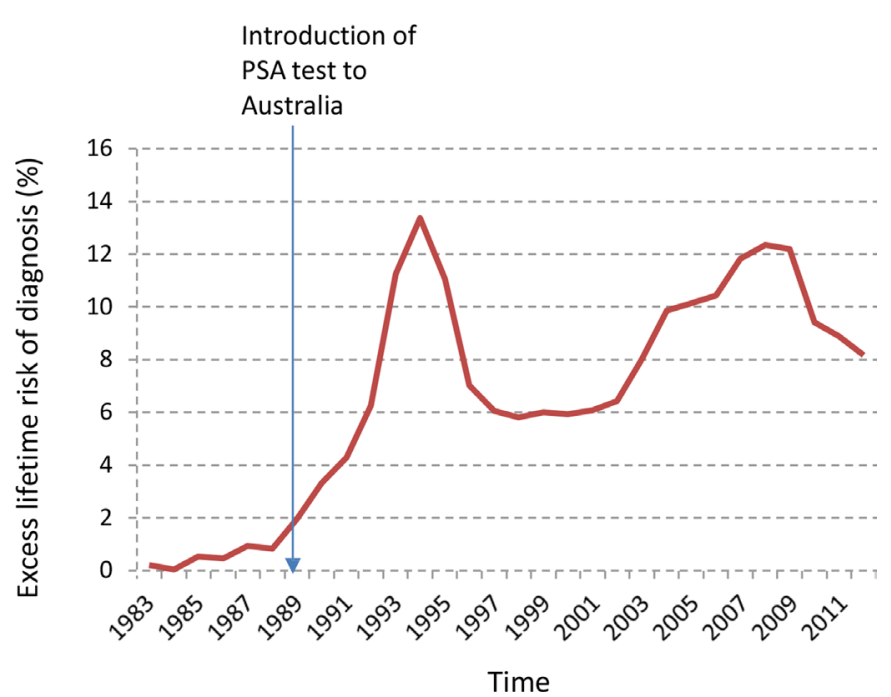

Figure 2 Excess lifetime risk of diagnosis of prostate cancer in Australia from 1982 to 2012. Estimated excess lifetime risk of diagnosis $=$ (adjusted lifetime risk of diagnosis in year after 1982-adjusted lifetime risk of diagnosis in 1982). To allow for the compensatory drop expected with the earlier detection of non-overdiagnosed cancers through screening, a sufficient time is needed after the introduction of screening before calculation of excess life risk of diagnosis provides a valid estimate of lifetime risk of overdiagnosis. For prostate cancer in Australia, the excess lifetime risk of prostate cancer for calendar years after 2009 (20 years after PSA introduced) may indicate lifetime risk of overdiagnosis. PSA, prostatespecific antigen.

population from 20 years after introduction of PSA (ie, 2009 onwards). The maximal estimated lifetime risk of overdiagnosis was around 12.2\% (11.6\%-12.8\%) in 2009 (one in nine men would have an overdiagnosed cancer in their lifetime) (online supplementary table 2). There was a subsequent decline in risk of overdiagnosis with an estimate in the most recent year (2012) of around $8.2 \%$ $(7.6 \%-8.7 \%)$ (1 in 13 men overdiagnosed with cancer). Calculated as the proportion of cancers diagnosed over a lifetime in unscreened men, we estimate that $71 \%$ of prostate cancers may be overdiagnosed (8\% lifetime risk of overdiagnosed cancer in 2012/11.5\% adjusted lifetime risk of prostate cancer in 1982). Calculated as the proportion of cancers diagnosed over a lifetime in screened men, we estimate that $41 \%$ of prostate cancers may be overdiagnosed ( $8 \%$ lifetime risk of overdiagnosed cancer $/ 19.6 \%$ adjusted lifetime risk of prostate cancer in 2012).

\section{Checking assumptions and sensitivity analysis using birth cohort data}

To check the assumption that screening rates were established, and the extent to which older men in recent years had previously been exposed to screening activities, we examined age-specific rates of PSA testing. Annual data on PSA testing were obtained from the MBS, which lists Medicare services subsidised by the Australian government; specifically, we examined claims made for MBS item 66655 ('PSA used only for screening purposes', data available only from 2001). ${ }^{25}$ Although these trends show some increases and decreases across all age groups, PSA screening appears to be well established in the population by 2012 (online supplementary figure 1).

The assumption that screening is well established is necessary when using cross-sectional data to calculate excess lifetime risk. However, if birth cohort data are used instead, this assumption may be relaxed, as earlier exposure to screening will be accounted for in the observed incidence rates at younger ages for the cohort. In figure 3, we can see that there are some differences in the age-specific prostate cancer incidence for different birth cohorts, which is likely to reflect different exposures to PSA screening. As a supplement to our main analysis using cross-sectional data, we conducted a sensitivity analysis using the age-specific incidence and mortality data for two birth cohorts of men to calculate excess lifetime risk. The older birth cohorts were men born in 1912, and these men were mostly unexposed to PSA up to age 80 years (PSA was first introduced into Australia when they were 79 years old). The younger birth cohorts were men born in 1932, and these men were exposed to PSA screening from approximately age 57 to 80 years. Where the relevant age-specific incidence, mortality or population data were not available, we used available rates from years that were closest in time to the missing rates. As age-specific incidence rates were only available from 1982 onwards, we had to use the same age-specific incidence rates for both birth cohorts up until age 70 . We used the same age-specific mortality rates for both birth cohorts to allow for changes in competing risk; these were based on those for the 1932 birth cohort with rates (with age-specific rates from 1972 used for age <40 years, as this is the earliest data available).

The cumulative risk of a prostate cancer diagnosis by age 80 for men born in 1912, adjusted for mortality rates observed for the 1932 birth cohort, was $5.1 \%$. The cumulative risk of a prostate cancer diagnosis by age 80 for men born in 1932 was $8 \%$, thus, there was an excess cumulative risk of $2.9 \%$. This corresponds to an overdiagnosis rate of $2.9 / 8.0=36.3 \%$, as a proportion of cancers in a screened population-similar to our estimated overdiagnosis rate of $41 \%$ calculated using the cross-sectional data for 2012 vs 1982.

From figure 3, we can see that the age-specific estimates for the 1932 birth cohort (second most left-hand column) tend to be lower than the age-specific 2012 data used for the cross-sectional analysis (open circles). This is especially the case for men in the age groups under 60 years who are largely unscreened in the 1932 birth cohort. Further, as noted above, because of missing data for age-specific incidence prior to 1982, we had to use the same rates both screened and unscreened cohorts up to age 70 and lifetime risk for the 1912 birth cohort is likely to have been overestimated. Therefore, our estimate of excess cumulative risk of a prostate cancer diagnosis using the 1932 vs 1912 birth cohorts is likely to underestimate overdiagnosis for contemporary populations of men who are exposed to PSA testing from age 40 or younger. 


\section{Prostate cancer incidence rate by birth cohort (log scale)}

10000

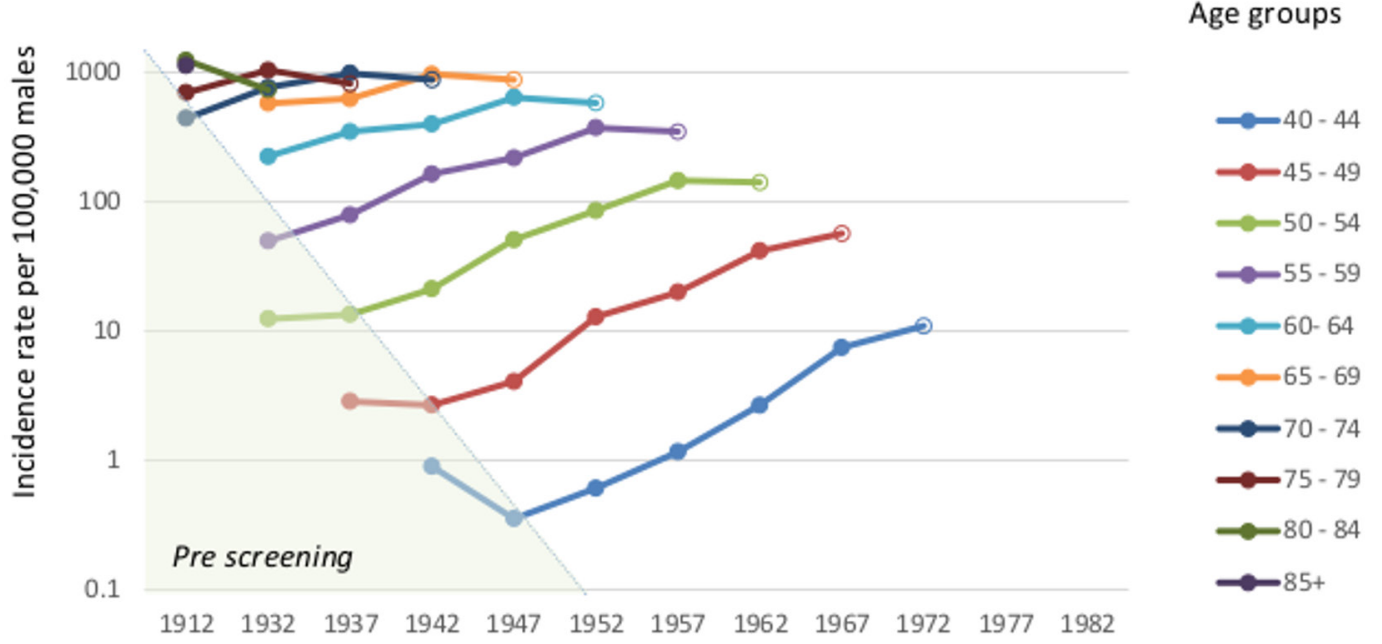

Birth Year

Figure 3 Age-specific prostate cancer incidence by birth cohort from 1982 to 2012. Age-specific incidence rates of prostate cancer by midyear of birth cohort, Australia 1982-2012. The years of birth on the horizontal axes in figure 3 indicate the midpoints of non-overlapping 5-year birth cohorts (except for the period 1912-1932). The points vertically above each midpoint year show that cohort's age-specific incidence rates (on the log scale). The data points corresponding to the incidence in 2012 used for calculating overdiagnosis using cross-sectional data are indicated by an open circle at the right-hand side of each age-specific incidence trajectory. The single incidence data point (white circle with a black outline) for 80-84 age group in 1932 birth year (741) overlaps very closely with the 70-74 age group incidence data point (dark blue) for 1932 (772) in the log scale. Therefore, it is not clearly visible. Source: Australian Institute of Health and Welfare data. ${ }^{21} 24$

\section{DISCUSSION}

Our estimate that $41 \%$ of screen-detected cancers are overdiagnosed is comparable to previously published estimates derived from data from follow-up reports after prostate cancer screening RCTs. The most recent estimate of overdiagnosis from the 13 years follow-up report of the European Randomised Study of Screening for Prostate Cancer was around $43 \%$ of screen-detected cancers ${ }^{26}$ while the previous estimates based on the same trial varied from $50 \%$ to $67 \%{ }^{27-30}$ A recent review by United States Preventive Services Task Force also reported that 20\%-50\% of screen-detected prostate cancers were overdiagnosed. ${ }^{19}$

Compared with the pre-PSA era, a number of factors are likely to have contributed to the excess incidence of prostate cancer observed following the introduction of PSA screening. ${ }^{31} 32$ These include: increased awareness of the disease, participation in screening activities, lowering of the histopathology threshold for diagnosis of cancer and interobserver disagreement on the histopathology diagnosis of cancer, ${ }^{33}$ and importantly discrepancy between a histopathology diagnosis of cancer and actual clinical significance/natural history of the lesion if left untreated. ${ }^{35} 36$ These factors help to explain how availability of PSA screening may result in overdiagnosis, but are not alternative hypotheses for the apparent overdiagnosis found in our results. The only alternative explanation to overdiagnosis that is consistent with the data is a true change in clinically important prostate cancer incidence due to changes in risk factors over time, but such a large increase in underlying risk seems unlikely.

\section{Challenges to the method}

Using life tables based on annual (cross-sectional) data to calculate lifetime risk of cancer overdiagnosis has similar applicability issues for the hypothetical cohort as when using this method to calculate lifetime risk of cancer including the limitations that come with using aggregated population data. Additionally, it has the requirement that the data must capture potential benefits in later life of early cancer detection at a younger age. This means that screening needs to be established for a period at least as long as the average screening lead time of non-overdiagnosed cancers. This requirement may be relaxed where birth cohort (longitudinal) data are used, but there may often be an insufficiently long period of available data for accurate estimates, as was the case in our example. And regardless of whether annual or birth cohort data are used, we are still reliant on aggregated population data, and we do not know the lifetime screening experience of individual people.

The method also assumes that there are no important changes in risk factors for the cancer which would change the true age-specific incidence. While this may be reasonable for prostate cancer, it may not be for other cancers. For example, for lung cancer, we would need to consider changes in the rates of smoking over time, and 
the time lag we would expect for consequent changes in underlying (non-overdiagnosed) lung cancer rates to be observed. Development of an additional modelling step to the life table method may account for such underlying temporal changes in risk factors.

The introduction of new technologies or screening protocols (eg, greater number of prostate cores sampled with prostate biopsy after a positive PSA test result) could increase detection rates in more recent calendar years compared with previous years. If some of this is beneficial detection of clinically significant cancers, then using cross-sectional data may overestimate the extent of overdiagnosis. Conversely, there is likely to have been overdiagnosed cancers included in the unscreened estimates from 1982, due to incidental diagnosis of indolent cancers in Trans Urethral Resections of the Prostate and other surgical procedures, which would lead to an underestimation of overdiagnosis.

\section{Utility of the method}

Despite some limitations, there are clear advantages to the life table method, especially the relaxation of the requirement that estimates must be adjusted for lead times of non-overdiagnosed cancers. The method is also relatively simple to apply using freely available statistical software and publicly available administrative datasets. It may be used to estimate the extent of overdiagnosis in populations where data from RCTs are not available. While we are not suggesting that this method should replace other methods, it may be used in combination with other methods to triangulate the likely extent of cancer overdiagnosis to produce a plausible upper and lower range of estimates, and to assess whether overdiagnosis is large or small. ${ }^{37}$

The analysis of long-term follow-up after screening RCTs remains the gold-standard method for estimating the effects of screening, both beneficial mortality reduction and overdiagnosis, but requires very long-term follow-up beyond the end of trial, and measurement of post-trial screening of participants which is currently not done. In the meantime, the life table method for estimating lifetime risk of overdiagnosis described in this paper is a valuable addition to existing methods for estimating overdiagnosis.

\section{CONCLUSION}

Population data for cancer incidence, cancer mortality and all-cause mortality rates may be used to estimate the lifetime risk of cancer overdiagnosis using methods commonly employed to estimate the lifetime risk of cancer diagnosis. Using cross-sectional data, the method may give valid estimates in populations where screening has been established long enough for the benefits from the early detection of non-overdiagnosed cancer at a younger age to be realised in older age groups (established longer than the average lead time). Birth cohort data may also be used and may be increasingly informative as cohorts exposed to screening at a younger age are growing older. This novel method may usefully complement existing methods to estimate overdiagnosis.

\section{Author affiliations}

${ }^{1}$ Center for Research in Evidence Based Practice, Bond University, Gold Coast, Queensland, Australia

${ }^{2}$ School of Medicine, Griffith University, Sunshine Coast, Queensland, Australia ${ }^{3}$ Australian Centre for Public and Population Health Research, Faculty of Health, University of Technology Sydney, Sydney, New South Wales, Australia

${ }^{4}$ School of Public Health, University of Sydney, Sydney, New South Wales, Australia

Acknowledgements We thank Professor Les Irwig for his valuable comments on an earlier draft of the manuscript.

Contributors TP is a medical doctor completing her $\mathrm{PhD}$ on overdiagnosis of prostate cancer at Centre for Research in Evidence-based Practice (CREBP), Bond University, Australia and a senior lecturer in public health at School of Medicine, Griffith University, Australia. AH is a biostatistician and professor of public health, faculty of health, University of Technology Sydney. JD is a professor of clinical epidemiology in the Centre for Research in Evidence-Based Practice in Bond University and a general practitioner. PG is a professor of evidence-based medicine, the director of the Centre for Research in Evidence-Based Practice in Bond University and a general practitioner. $\mathrm{KB}$ is a senior lecturer in clinical epidemiology in the School of Public Health at the University of Sydney. KB conceived the ideas, developed them with all coauthors and edited the paper. TP retrieved data from Australian Institute of Health and Welfare, did the analyses, wrote the first draft, edited the paper and is guarantor. AH, JD and PG developed the ideas and edited the paper.

Funding The authors have received funding from the Australian National Health and Medical Research Council (NHMRC Fellowship No. 1080042, Centres of Research Excellence grant No. 1104136: Creating sustainable health care: ensuring new diagnostics avoid harms, improve outcomes and direct resources wisely and Program grant No. 1113532: Using healthcare wisely: reducing inappropriate use of tests and treatments).

Disclaimer The funders had no role in design and conduct of the study; collection, management, analysis and interpretation of the data; and preparation, review or approval of the manuscript.

Competing interests None declared.

Patient consent for publication Not required.

Provenance and peer review Not commissioned; externally peer reviewed.

Data sharing statement Additional data available on contacting corresponding author (TP) through email (thanindu@gmail.com).

Open access This is an open access article distributed in accordance with the Creative Commons Attribution Non Commercial (CC BY-NC 4.0) license, which permits others to distribute, remix, adapt, build upon this work non-commercially, and license their derivative works on different terms, provided the original work is properly cited, appropriate credit is given, any changes made indicated, and the use is non-commercial. See: http://creativecommons.org/licenses/by-nc/4.0/.

\section{REFERENCES}

1. Bell KJ, Del Mar C, Wright G, et al. Prevalence of incidental prostate cancer: a systematic review of autopsy studies. Int J Cancer 2015;137:1749-57.

2. Gulati R, Feuer EJ, Etzioni R. Conditions for valid empirical estimates of cancer overdiagnosis in randomized trials and population studies. Am J Epidemiol 2016;184:140-7.

3. Carter JL, Coletti RJ, Harris RP. Quantifying and monitoring overdiagnosis in cancer screening: a systematic review of methods. BMJ 2015;350:g7773.

4. Ripping TM, Ten Haaf K, Verbeek ALM, et al. Quantifying overdiagnosis in cancer screening: a systematic review to evaluate the methodology. J Natl Cancer Inst 2017;109:djx060-djx.

5. Jacklyn G, McGeechan K, Houssami N, et al. Overdiagnosis due to screening mammography for women aged 40 years and over. Cochrane Database Syst Rev 2018;22.

6. Baker SG, Prorok PC, Kramer BS. Lead time and overdiagnosis. J Natl Cancer Inst 2014;106:dju346-dju. 
7. Zahl PH, Jørgensen KJ, Gøtzsche PC. Lead-time models should not be used to estimate overdiagnosis in cancer screening. J Gen Intern Med 2014;29:1283-6.

8. Zahl PH, Jørgensen KJ, Gøtzsche PC. Overestimated lead times in cancer screening has led to substantial underestimation of overdiagnosis. Br J Cancer 2013;109:2014-9.

9. Puliti D, Duffy SW, Miccinesi G, et al. Overdiagnosis in mammographic screening for breast cancer in Europe: a literature review. J Med Screen 2012;19(Suppl 1):42-56.

10. Duffy SW, Lynge E, Jonsson $\mathrm{H}$, et al. Complexities in the estimation of overdiagnosis in breast cancer screening. Br J Cancer 2008;99:1176-8.

11. Etzioni R, Gulati R, Mallinger L, et al. Influence of study features and methods on overdiagnosis estimates in breast and prostate cancer screening. Ann Intern Med 2013;158:831-8.

12. Duffy SW, Parmar D. Overdiagnosis in breast cancer screening: the importance of length of observation period and lead time. Breast Cancer Res 2013;15:R41.

13. Biesheuvel C, Barratt A, Howard K, et al. Effects of study methods and biases on estimates of invasive breast cancer overdetection with mammography screening: a systematic review. Lancet Oncol 2007;8:1129-38.

14. Cancer Research UK. Breast cancer statistics. 2017 http://www. cancerresearchuk.org/health-professional/cancer-statistics/statisticsby-cancer-type/breast-cancer - heading-Zero (5.10.2017).

15. Fay MP, Pfeiffer R, Cronin KA, et al. Age-conditional probabilities of developing cancer. Stat Med 2003;22:1837-48.

16. Surveillance Research Program Statistical Methodology and Applications. DevCan: Probability of Developing or Dying of Cancer Software, Version 6.7.5. 2012 http://surveillance.cancer.gov/ devcan/.

17. Pepe MS, Mori M. Kaplan-Meier, marginal or conditional probability curves in summarizing competing risks failure time data? Stat Med 1993;12:737-51.

18. Marmot MG, Altman DG, Cameron DA, et al. The benefits and harms of breast cancer screening: an independent review. Br J Cancer 2013;108:2205-40.

19. Agency for Healthcare Research and Quality. U.S. Department of Health and Human Services. Overdiagnosis in Prostate Cancer Screening Decision Models: A Contextual Review for the U.S. Preventive Services Task Force: Agency for Healthcare Research and Quality, 2017.

20. Smith DP, Armstrong BK. Prostate-specific antigen testing in Australia and association with prostate cancer incidence in New South Wales. Med J Aust 1998;169:17-20.
21. Australian Institute of Health and Welfare. Australian Cancer Incidence and Mortality (ACIM) books: Prostate cancer. Canberra: Australian Institute of Health and Welfare, 2016.

22. Department of Human Services. Medicare Item Reports Australia: Australian Government;. 2016 http://medicarestatistics. humanservices.gov.au/statistics/mbs_item.jsp (5 Oct 2017).

23. Australian Government. Australian Institute of Health and Welfare. Australian Cancer Database, Quality Statement. 2017 http://meteor. aihw.gov.au/content/index.phtml/itemld/687104.

24. Australian Institute of Health and Welfare. General Record of Incidence of Mortality (GRIM) workbooks:All causes combined, 2013.

25. Department of Human Services. Medicare Item Reports Australia: Australian Government. 2015 http://medicarestatistics. humanservices.gov.au/statistics/mbs_item.jsp (10 Oct 2018).

26. Heijnsdijk EA, Wever EM, Auvinen A, et al. Quality-of-life effects of prostate-specific antigen screening. $N$ Engl J Med 2012;367:595-605.

27. Welch HG, Black WC. Overdiagnosis in cancer. J Natl Cancer Inst 2010;102:605-13.

28. Heijnsdijk EA, der Kinderen A, Wever EM, et al. Overdetection, overtreatment and costs in prostate-specific antigen screening for prostate cancer. Br J Cancer 2009;101:1833-8.

29. Schröder FH, Hugosson J, Roobol MJ, et al. Prostate-cancer mortality at 11 years of follow-up. N Engl J Med 2012;366:981-90.

30. Draisma G, Boer R, Otto SJ, et al. Lead times and overdetection due to prostate-specific antigen screening: estimates from the European Randomized Study of Screening for Prostate Cancer. J Natl Cancer Inst 2003;95:868-78.

31. Pathirana T, Clark J, Moynihan R. Mapping the drivers of overdiagnosis to potential solutions. BMJ 2017;358:j3879.

32. Loeb S, Bjurlin MA, Nicholson J, et al. Overdiagnosis and overtreatment of prostate cancer. Eur Urol 2014;65:1046-55.

33. Elmore JG, Longton GM, Carney PA, et al. Diagnostic concordance among pathologists interpreting breast biopsy specimens. JAMA 2015;313:1122-32

34. Elmore JG, Barnhill RL, Elder DE, et al. Pathologists' diagnosis of invasive melanoma and melanocytic proliferations: observer accuracy and reproducibility study. BMJ 2017;357:j2813.

35. Black WC. Overdiagnosis: an underrecognized cause of confusion and harm in cancer screening. J Natl Cancer Inst 2000;92:1280-2.

36. Barratt A, Irwig L, Glasziou P, et al. Users' guides to the medical literature: Xvii. How to use guidelines and recommendations about screening. JAMA 1999;281:2029-34.

37. Baker SG, Prorok PC, Kramer BS. Editorial: challenges in quantifying overdiagnosis. J Nat/ Cancer Inst 2017;109:djx064-djx. 